\title{
LA REGULACIÓN DE LA FORMACIÓN Y EL TRABAJO DOCENTE: UN ANÁLISIS CRÍTICO DE LA "AGENDA EDUCATIVA” EN AMÉRICA LATINA
}

Myriam FeLdFeber

\begin{abstract}
RESUMEN: Este trabajo analiza las discusiones en materia de políticas de formación y trabajo docente que se han venido incorporando en las agendas educativas de la Región en los últimos años. Sostenemos que las nuevas formas de regulación propuestas son modos de imposición heterónomos contrarios a los objetivos que formalmente se proponen en materia de profesionalización y autonomía de los docentes.
\end{abstract}

Palabras clave: Regulación educativa. Profesionalización docente. Formación y trabajo docente. América Latina.

REGULATION OF TEACHER TRAINING AND WORK:

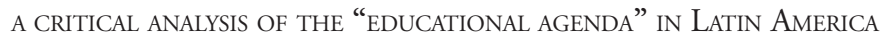
ABSTRACT: This paper analyses the discussions about teacher training and work that have been gaining momentum in the educational agendas of the region in recent years. It argues that the proposed forms of regulation are heteronomous modes of imposition that are contradictory with their declared goals in terms of professionalization and teacher autonomy.

Key words: Educational regulation. Teacher professionalization.

Teacher training and work. Latin America.

Profesora de las Facultades de Filosofía y Letras y Ciencias Sociales e investigadora del Instituto de Investigaciones en Ciencias de la Educación, Universidad de Buenos Aires. E-mail: mfeldfeb@filo.uba.ar 


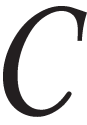

arlos Fuentealba, trabajador docente, fue asesinado el 4 de abril de 2007 en la provincia de Neuquén, en Argentina, mientras reclamaba por mejoras salariales. Un Estado provincial, que utiliza la fuerza policial para reprimir la demanda social, asesinó a un hombre que luchaba por la defensa de la escuela pública. Enseñaba física y química en escuelas secundarias que reciben a adolescentes y jóvenes en condición de pobreza. La periodista Sandra Russo se preguntaba por los alumnos de Carlos Fuentealba y, recordando el fotógrafo brasileño Sebastián Salgado, señalaba:

(...) si yo fuera Salgado, iría a Neuquén y retrataría a cada uno de esos chicos. Si tuviera el talento de Salgado, lo usaría para que en esos retratos fuera visible la ausencia del maestro. ¿Qué sueños acompañaba Fuentealba? ¿Qué lección marcará a fuego esas aulas en las que los hijos de los pobres intentan todos los días quebrar el destino que tienen reservado? ¿Qué tipo de extraña melancolía se adhiere a esos chicos, como a los otros chicos que retrató Salgado? ¿Cómo se reflejará en sus miradas el asombro infinito por el asesinato del maestro? (Diario, 10 abr. 2007, p. 12)

El análisis de las políticas en materia de formación y trabajo docente no puede realizarse al margen de las condiciones efectivas en las que los maestros y profesores desarrollan cotidianamente su tarea. Desde esta perspectiva es posible observar la distancia existente entre los temas que se incluyen en las agendas, que se discuten a nivel global y regional impulsadas por organismos internacionales y regionales, por grupos de trabajo promovidos por estos organismos y por los técnicos ministeriales, y las condiciones objetivas y subjetivas de los docentes y de las prácticas educativas en la coyuntura de cada uno de los países de la Región.

Tampoco puede realizarse este análisis al margen de las profundas transformaciones económicas, sociales, políticas y culturales de las últimas décadas, que cuestionan los sentidos de la escolarización y el rol de los docentes. Entre otras transformaciones podemos señalar: una nueva estructura de poder internacional propia de la etapa actual de reestructuración del capitalismo; los procesos de reforma de los Estados nacionales y las nuevas funciones que estos asumen en el contexto de la globalización; los procesos de individualización y polarización social que multiplican los ejes de la desigualdad; la crisis del modelo de la sociedad salarial y la flexibilización de las relaciones laborales; los cambios en la estructura social y en el modelo productivo; la mundialización de la 
La regulación de la formación y el trabajo docente...

cultura; el impacto de las nuevas tecnologías de la información y al comunicación; la crisis institucional de la escuela y del proyecto moderno que orientó la institucionalización de sistemas educativos centralizados y las nuevas demandas que se le formulan a las escuelas y a los docentes desde el paradigma de la sociedad del conocimiento. ${ }^{1}$

En este trabajo, analizamos las discusiones en materia de políticas destinadas a regular la formación y el trabajo de los docentes que se han venido incorporando de modo general en las agendas educativas de la Región en los últimos años, partir de documentos promovidos por organismos regionales e internacionales, consultores, y grupos de trabajo financiados por dichos organismos. Sostenemos que las nuevas formas de regulación que se proponen constituyen modos de imposición heterónomos, que contradicen los objetivos que formalmente se proponen alcanzar en materia de profesionalización y autonomía de los docentes.

A diferencia de los modos de regulación típicamente burocráticos que apelaban al docente como categoría social homogénea, las nuevas formas post-burocráticas que buscan regular la formación y el trabajo docente se dirigen a los docentes como individuos libres, que construyen su propia carrera y destino profesional. Se intenta pasar así del control burocrático característico de las sociedades disciplinarias (Deleuze, 1991) a modelos que discursivamente proponen la autonomía del docente como profesional y la autorregulación, pero que condicionan dicha autonomía a través de medidas basadas en la lógica del mercado: redefinición de la carrera docente a partir de la flexibilización de las relaciones laborales, salario basado en el mérito, premio al desempeño, incentivos para atraer a "los mejores" a la profesión, evaluación basada en "reglas objetivas", mecanismos de acreditación y definición de estándares a nivel nacional e internacional.

La idea que subyace es que el docente debe hacerse a sí mismo en un "mercado profesional", cuyas reglas de funcionamiento están definidas por el Estado. En el mercado, el docente debe ofrecer su fuerza de trabajo a cambio de un salario que esté en función de su "productividad", y como profesional se ve obligado a capacitarse y obtener titulaciones para cumplir con los requisitos que le permitan permanecer en el mercado y ascender en su carrera laboral y profesional.

La incipiente recuperación de la centralidad de la política frente a la lógica economicista y tecnocrática hegemónica en los '90, en general, 
no ha logrado impactar en los modos de construcción política hacia el sector docente. Si bien comienza a observarse un desplazamiento de las demandas de profesionalización a modelos que proponen el desarrollo profesional, los docentes continúan siendo el "objeto de la política” en lugar de "profesionales participantes en ella" (Angus, 1994, apud Whitty, Power y Halpin, 1999, p. 86).

Los temas que integran la agenda educativa y que circulan a través de los organismos regionales e internacionales, de los consultores y de los grupos de trabajo, y de las fundaciones que se expandieron en los ' 90 , se articulan en torno a las demandas de profesionalización y autonomía de los docentes; las políticas en materia de formación continua y desarrollo profesional; la definición de nuevas carreras laborales; el establecimiento de sistemas de evaluación de los docente; la introducción de mecanismos para volver "atractiva" la profesión y atraer "jóvenes calificados" y el establecimiento de nuevos criterios para definir las escalas salariales en base al mérito y a la responsabilidad por los resultados. Estas medidas comienzan a funcionar como nuevos dispositivos para gobernar la educación. ${ }^{2}$ Si bien estas nuevas modalidades de gobierno de la vida social y de la conducta individual pueden abrir nuevas posibilidades reflexivas y creativas para la intervención del sujeto en la gestión de sus propias opciones vitales, al mismo tiempo se están inaugurando nuevos regímenes de sujeción heterónoma (De Marinis, 2005).

Esta "agenda globalmente estructurada para la educación” no se traduce en las políticas públicas de cada uno de los países de la Región. De acuerdo con Dale (2007), la globalización, que representa un cambio cualitativo en la naturaleza de las relaciones nacionales y supranacionales, no implica necesariamente una mayor homogeneidad en las políticas o en las prácticas educativas. Por lo tanto, la naturaleza e impacto de los efectos de la globalización varían enormemente entre los diferentes países, de acuerdo con la posición que ocupan dentro de la economía global y con sus propias estructuras económicas y de poder internas. Sin embargo, las recomendaciones de política de los organismos regionales e internacionales buscan imponerse más allá de las historias y especificidades de los diferentes sistemas educativos, de modo tal de instalar temas en las agendas sobre los cuales se discute su forma de implementación, pero no su incorporación como política pública. En este sentido, es elocuente la implementación de sistemas nacionales e internacionales 
La regulación de la formación y el trabajo docente...

de evaluación de la calidad: se discute cómo definir e implementar estos sistemas, pero no es materia de debate su propia existencia, aún cuando su impacto en términos de mejora de la calidad dista de ser evidente. En este sentido cabe preguntarse cómo estos temas logran condicionar las prioridades de política de los gobiernos nacionales.

Las reformas en materia de formación y trabajo docente se inscriben dentro de las nuevas propuestas de regulación y control social que tienen lugar en el ámbito de la enseñanza. Estos cambios suponen, entre otras cuestiones, la aprobación de una nueva legislación que gobierna la formación del profesorado, la creación de nuevas instituciones y modelos de certificación o habilitación para la función docente (Popkewitz y Pereyra, 1994, p. 16).

Los docentes - como categoría social cada vez más heterogénea y que presenta crecientes grados de desigualdad (Tenti Fanfani, 2005) son visualizados como parte del problema de la calidad a la vez que de la solución. Hace tiempo que el sector docente es señalado como responsable por el deterioro de la calidad educativa, hecho que ha ejercido gran influencia en el reconocimiento público y el status atorgado a la enseñanza (Burbules y Densmore, 1992). El informe "A Nation at Risk", elaborado a pedido del Presidente Reagan en EEUU, en 1983, criticaba la mediocridad de la escuela norteamericana y atribuía parte de la responsabilidad a la incompetencia de los profesores. El Informe formulaba recomendaciones de política orientadas a "mejorar la preparación de los maestros o a hacer que la profesión docente sea más satisfactoria y respetada". Entre ellas se señalaba que los salarios de los docentes deben incrementarse y deben ser competitivos, guiados por las señales del mercado y basados en la performance. Las decisiones sobre el salario, la promoción, el cargo y la retención deben estar atadas a un sistema de evaluación efectivo que incluya revisión de pares, de manera que los mejores docentes puedan ser recompensados, los docentes "promedio" incentivados, y los peores, o bien mejorados o bien despedidos.

La idea que subyace a este informe, como a muchos de los documentos que analizamos en este trabajo y que han tenido difusión en los últimos años, es que sólo un docente "disciplinado" por las reglas del mercado puede ser agente de la mejora de la educación. El problema es el docente colectivo, sindicalizado, burocratizado y más preocupado por 
reivindicaciones gremiales que por la enseñanza; la solución es el docente individual, emprendedor y creativo, que busca capacitarse continuamente para progresar en su carrera y mejorar su remuneración.

Tras estas propuestas de modificación de la carrera laboral en base a incentivos y evaluación de los docentes, subyace un modelo del trabajador autónomo que implica un matiz capitalista como empresario de sí mismo; por eso mientras el capitalismo temprano se orientó a explotar el trabajo, el contemporáneo explota la responsabilidad individual, demandando a los individuos soluciones biográficas a problemas que son estructurales (Beck, 2004).

Las demandas de profesionalización y el control de la autonomía en el trabajo docente

La mayor parte de los ministerios y secretarías de educación de la Región han incorporado, en sus agendas de política, propuestas en materia de profesionalización de los docentes. Estas incluyen desde los criterios de selección y reclutamiento de estudiantes de magisterio, pasando por los programas de formación inicial y permanente, los mecanismos de ingreso al sistema educativo, la estructura y dinámica de la carrera docente, el sistema de incentivos, hasta los mecanismos de evaluación del desempeño docente (Tenti Fanfani, 2004).

La inclusión de las discusiones en torno a la profesionalización de la docencia en la agenda pública no constituye un fenómeno novedoso. Sin embargo, han adquirido un nuevo impulso a partir de la década de los '90 con la elaboración de informes y estudios impulsados y financiados por organismos internacionales y regionales y la conformación de grupos de trabajo destinados a discutir y difundir las denominadas políticas de "profesionalización" de los docentes.

La profesionalización y el protagonismo de los educadores constituyen uno de los ejes de política de la propuesta de CEPAL y UNESCO, "Educación y conocimiento: ejes de la transformación productiva con equidad", publicada en 1992, que ejerció una notable influencia en las discusiones de política de la Región. El trabajo propone establecer nuevos mecanismos de regulación política donde el Estado, a la vez que otorga mayor autonomía a los establecimientos para el mejoramiento de la calidad, dispone de medios eficaces para evaluar el cumplimiento de 
La regulación de la formación y el trabajo docente...

las metas así como resguardar las orientaciones globales del sistema y su equidad.

El informe señala que existen básicamente dos exigencias en relación con un sistema eficiente de recursos humanos: el compromiso con una educación de calidad y la capacidad para administrar en forma autónoma y responsable los establecimientos y recursos a su cargo, profesionalizando urgentemente los cargos docentes. Para ello se propone atender a los procesos de reclutamiento, formación y capacitación de los docentes, revisar el sistema de remuneraciones y establecer incentivos en función de la productividad. Propone dar libertad a los establecimientos educativos para determinar total o parcialmente las remuneraciones de sus maestros, en función de su propio proyecto educativo. La evaluación del rendimiento de los docentes se inscribe dentro del objetivo de propiciar una gestión institucional responsable, de modo de garantizar la existencia de un eficaz mecanismo de información y evaluación del rendimiento escolar y docente, para que "los usuarios puedan demandar" que se eleve la calidad de enseñanza, los establecimientos mejoren su desempeño y las autoridades puedan centrar su acción donde los rendimientos sean más bajos y afecten la equidad del sistema en su conjunto.

La profesionalización de los docentes constituye el tema del Grupo de Trabajo sobre "Desarrollo Profesional Docente" (GTD) ${ }^{3}$ - inicialmente denominado Grupo de Trabajo sobre Profesionalización Docente -, creado en abril de 2004 en el marco del Programa de Promoción de la Reforma Educativa en América Latina y el Caribe (PREAL), ${ }^{4}$ el cual se propone como objetivo "analizar las políticas dirigidas a los docentes en distintos países latinoamericanos, promoviendo un diálogo regional y difundiendo experiencias exitosas en este campo". El GTD centra su acción en la formación inicial y continua, la situación laboral y la gestión institucional de la docencia. Las actividades del Grupo incluyen la divulgación de un boletín mensual, la elaboración de estudios e informes con recomendaciones de política, la compilación de artículos y documentos relevantes, la difusión de noticias y eventos de interés, a través de su página web, y la realización de encuentros regionales y seminarios internacionales.

Los sentidos que asume la profesionalización constituyen un objeto de lucha, donde confluyen múltiples actores colectivos e intereses 
diversos que intentan direccionar las políticas en materia de formación y trabajo docente. Lo que está en juego es el control de la autonomía en el trabajo (Lang, 2006; Tenti Fanfani, 2006). A partir de la década de los ' 90 , las políticas, en materia de autonomía y profesionalización de la docencia, estuvieron en su gran mayoría orientadas por una racionalidad tecnocrática y una retórica modernizadora. Buscaron imponerse desde una lógica "top down": reformas diseñadas en las esferas centrales de gobierno con alta presencia de los equipos técnicos y escasa o nula participación de los docentes, estableciendo lo que los docentes debían conocer y hacer para "profesionalizarse" (Feldfeber, 1999). En general,

(...) tendieron a proponer mayores dosis de "autonomía" y la accountability de los docentes (apelando a su creatividad, compromiso, liderazgo, trabajo en equipo, por proyectos, etcétera); al mismo tiempo, desplegaban un conjunto de dispositivos de medición de calidad de los resultados del aprendizaje (evaluación de rendimiento mediante pruebas estandarizadas), definición de mínimos curriculares y estándares de aprendizaje, evaluación de la calidad profesional de los docentes (mediante la identificación de "competencias" pedagógicas), pago por rendimiento, entre otros. Dichos dispositivos constituían, en los hechos, mecanismos que reforzaban los controles externos sobre el trabajo de los docentes. (Tenti Fanfani, 2006, p. 136-137)

Lang (2006) señala que, tal como sucede con otros grupos profesionales, en la actualidad asistimos a la desaparición de las identidades docentes colectivas y uniformes. En un contexto de debilitamiento de la cohesión de los grupos y de incertidumbre en cuanto a sus misiones, las políticas de profesionalización constituyen necesariamente una fuente de polémica. De cierta manera, reproducen las tensiones que existen entre la autonomía y el control, características de estos grupos profesionales bajo la tutela del Estado, desde el origen de los mismos hasta el siglo XIX. Pero, al imponer la profesionalización sin el consentimiento de los actores, la misma puede poner en tela de juicio la propia índole del oficio, la elección y la naturaleza de las acciones profesionales y la actitud frente al oficio, elementos primordiales en la construcción de la autonomía de un grupo profesional (Lang, 2006, p. 116).

A su vez, el proceso de descalificación y desvalorización sufrido por los docentes en América Latina ha provocado cambios significativos en su identidad a la par que se han precarizado sus condiciones de trabajo (Oliveira, 2005). 
La regulación de la formación y el trabajo docente...

La remuneración y los incentivos como mecanismos de regulación: cómo atraer a jóvenes calificados

Atraer a los jóvenes "más calificados" o a "los mejores", establecer incentivos y modificar la carrera laboral constituyen parte de los principales mecanismos a través de los cuales se intenta regular la formación y el trabajo docente.

La Organización para la Cooperación y el Desarrollo Económico (OCDE, 2004, apud Torres, 2005), otro organismo internacional que busca influir en el diseño de políticas públicas, planteó una "estrategia global" para atraer, capacitar y conservar a profesores eficaces que incluye 5 puntos:

- Hacer de la enseñanza una opción profesional atractiva, lo que, por ejemplo, exige mejorar la imagen y el prestigio de esta carrera, así como "la competitividad de las remuneraciones" y de las condiciones de trabajo.

- Reforzar los conocimientos y las competencias de los docentes, lo que pasa por flexibilizar su formación inicial, adaptarla mejor a las necesidades de los centros escolares y reforzar su perfeccionamiento profesional a lo largo de su carrera.

- Reclutar, seleccionar y emplear a los mejores profesores posibles. Para ello hay que flexibilizar sus condiciones de empleo y dar a los centros de enseñanza más responsabilidades en la selección y gestión del personal.

- Conservar a los docentes de calidad, lo que pasa por la evaluación y recompensa de la eficacia pedagógica y por ofrecerles posibilidades de diversificar su trayectoria profesional.

- Hacer participar a los docentes en la elaboración de la política de educación. (Torres, 2005, p.93)

Para volver más atractiva la profesión y así poder reclutar a los mejores, se plantea la necesidad de aumentar los salarios. Sin embargo, algunos de los estudios (incluso los que proponen políticas más agresivas en términos de flexibilización salarial) concluyen que no necesariamente un incremento salarial tendría impacto en atraer y retener a "los mejores" maestros. Por ejemplo, uno de los trabajos financiados por el Banco 
Mundial compara los ingresos anuales de los maestros de 12 países latinoamericanos con los de otras profesiones "comparables", y concluye que si bien los maestros cobran menos, como trabajan menos horas, en promedio ganan más si se toma en consideración el salario por hora. A partir de esta constatación, argumenta que un incremento en el salario podría no tener impacto en el reclutamiento y la retención de mejores maestros. Además, propone que se deben dar más oportunidades para los maestros que quieran trabajar más horas y ganar más. Dos tercios de los maestros en los países estudiados son mujeres, cuyos ingresos constituyen un segundo ingreso familiar. Para el autor, esto abonaría la hipótesis de que los que eligen la docencia lo hacen por la menor carga horaria y por la flexibilidad de horarios (Liang, 1999).

En lo que respecta al establecimiento de incentivos en la carrera docente, un estudio apoyado por el Banco Interamericano de Desarrollo (BID), "Los maestros en América Latina: carreras e incentivos" (Diaz y Saavedra, 2000, p. 4) analiza los incentivos y factores institucionales que determinan la composición del cuerpo docente y que influyen en su desempeño profesional, factor crucial para el logro de la calidad educativa. Es por ello que se señala que

(...) un mayor rendimiento del maestro requiere cambiar las políticas de formación y de financiamiento educacional, así como otorgarle los incentivos adecuados. Siendo el desempeño docente el principal determinante de los resultados diferenciados de los alumnos, es crucial que cuidadosamente se planifique e implemente la selección de postulantes, la capacitación de los formadores de maestros y los estímulos para promover el aporte eficiente del profesorado en el aprendizaje. (Idem, ibid., p. 7)

De acuerdo con este estudio, la estabilidad laboral, que es un elemento atractivo de la carrera, es al mismo tiempo una muestra de los problemas de eficiencia en la administración de recursos humanos en las escuelas públicas. La docencia se configuró como una carrera en la que si bien los ingresos son bajos, son estables y seguros cuando se logra el nombramiento. También se afirma que es una carrera que no ofrece estímulos claros para innovar, actualizarse y tener un mejor desempeño. En la medida en que esto tiene un efecto sobre el perfil de individuo que va a preferir ejercer la carrera docente, se plantea la necesidad de revisar los incentivos a la profesión, la estabilidad laboral y la falta de autonomía de los directores para administrar el personal de 
La regulación de la formación y el trabajo docente...

sus instituciones - contratar y seleccionar a sus maestros y evaluar, sancionar o premiar el desempeño de su personal (idem, ibid., p. 53).

Un informe del Banco Mundial sobre Argentina señala que "el actual sistema de remuneraciones, basado exclusivamente en la antigüedad, es insuficiente para retener a los docentes, fomentar su desarrollo profesional o mejorar la enseñanza" (Vegas et al., 1999, p. 71). Por ello, proponen perfilar una "nueva carrera docente" que incluya incentivos por desarrollo profesional y por desempeño de la escuela.

Sin embargo, es importante destacar las contradicciones que subyacen a esta propuesta debido a que, mientras se propone el pago en función del mérito, se reconoce que "el sistema de pago basado en el mérito implementado en algunos países desarrollados no ha sido siempre exitoso, en parte por la dificultad de relacionar los resultados de la escuela con el comportamiento individual de los profesores" (Banco Mundial, 1999, p. 71).

De acuerdo con un estudio realizado en el marco del PREAL, la remuneración docente parece estar condicionando cada vez más el reclutamiento a la profesión. En efecto, como se indica en algunos estudios recientes, la docencia se ha transformado en una profesión que "no paga lo suficiente para atraer a los mejores candidatos", porque las estructuras de aumento salarial en la carrera son significativamente peores a las de otras profesiones. Esto hace que aquellos que ingresan a los institutos de formación tengan, en promedio, peor historial educativo que otros estudiantes que acceden a otros estudios más valorizados socialmente (Vaillant, 2004).

En síntesis, lo que se pone de manifiesto a partir del análisis de estos estudios es que existe una fuerte contradicción entre las evidencias, que no sostienen lo que se propone en términos de incentivos (y en algunos casos lo contradicen), y la insistencia en la necesidad de aplicar las mismas fórmulas. Además del dogmatismo que sustenta a gran parte de estos trabajos, esta contradicción demuestra un absoluto desconocimiento de las situaciones reales y concretas que atraviesan las escuelas y los docentes y de los modos en que se construyen los procesos de aprendizaje, aspectos que son invisibilizados en gran parte de estos enfoques.

Los interrogantes que constituyen el punto de partida de estos análisis, así como los supuestos que los orientan, también deben ser 
puestos en cuestión. Basta como ejemplo el documento de trabajo del GTD de PREAL, elaborado por Moura Castro e Ioschoe (2007), que se interrogan si la remuneración de los docentes es baja y si afecta la calidad de la enseñanza.

En cuanto a la definición de una nueva carrera docente, las propuestas de flexibilización laboral, que intentan avanzar sobre uno de los pocos empleos estables ligados a la sociedad salarial, orientan algunas de las propuestas de reforma, al tiempo que se plantea la necesidad de revisar una carrera organizada en torno al modelo burocrático y disciplinario que acompañó el desarrollo y expansión de los sistemas educativos modernos.

La flexibilización laboral, que constituye una de las consecuencias más visibles de la crisis del modelo de sociedad salarial (uno de los pilares del pacto social keynesiano desde la posguerra), conlleva profundas transformaciones en términos de constitución de identidad y garantía de los derechos de los trabajadores (Morgenstern de Finkel, 2000). Las condiciones que caracterizaron el modelo salarial fordista se han comenzado a modificar, en especial aquellas vinculadas con la estabilidad en el empleo y la existencia de una protección social ligada a la idea de derecho, lo que impacta fuertemente en la conformación de nuevas subjetividades. La desestructuración del carácter que se fue moldeando sobre la base de la rutina, la experiencia y las jerarquías que caracterizaban el modelo fordista (Sennett, 2000), más que fortalecer la autonomía de los individuos, puede generar un desorden vital en las personas que se encuentran arrojadas a la experiencia del riesgo, la inestabilidad y la incertidumbre, al tiempo que se desdibuja el concepto de interés común.

La evaluación del desempeño en la carrera laboral de los docentes

Un estudio sobre evaluación del desempeño y carrera profesional docente en 50 países de América y Europa (Murillo, 2006) sostiene que una de las actuales preocupaciones de los sistemas educativos de América y Europa es la de desarrollar sistemas de carrera docente y de evaluación del desempeño docente que contribuyan al desarrollo profesional de los maestros y profesores y, con ello, a la mejora de la calidad de la enseñanza. Sin embargo, se reconoce que la evaluación del desempeño 
La regulación de la formación y el trabajo docente...

docente es un tema altamente conflictivo, dado que en él se enfrentan intereses y opiniones de los políticos y administradores, de los docentes y sus sindicatos, y de los estudiosos sobre la materia.

El estudio destaca el caso de Finlandia, país que se ha convertido en estos años en el símbolo de la calidad de la educación, donde no sólo no existe evaluación externa de docentes o centros, sino que es un tema que ni siquiera está en debate. El sistema educativo finlandés se basa en la confianza sobre el docente y su profesionalidad, así como en el buen hacer de los centros educativos. El estudio afirma que

(...) es posible un sistema educativo de calidad sin un sistema de evaluación externa del desempeño docente. En ese caso, se sustituyen la presión y los estímulos externos por una confianza en la profesionalidad de los docentes. Y los centros escolares son el eje básico para la mejora de la educación. (Murillo, 2006, p. 29)

También señala que existe una generalizada opinión crítica de los estudiosos e investigadores acerca de las limitaciones de la evaluación. Sin embargo, y en forma contradictoria con las afirmaciones anteriores, propone que

(...) si se desea mantener e incrementar la motivación de los profesionales de la educación, reconociendo el buen trabajo de los docentes mediante un incremento salarial o la promoción en el escalafón docente, es necesario contar con un sistema que discrimine el buen desempeño docente del que no lo es tanto. Así, es necesario contar con un sistema de evaluación del desempeño profesional. (Idem, ibid., p. 30)

Se insiste en que dicho sistema debe estar aceptado por la comunidad educativa, fundamentalmente por los docentes, y ser impecable técnicamente y en que existe el riesgo de que el docente pueda caer en la tentación no de desarrollar bien su trabajo para cumplir con los elementos que son reconocidos en la evaluación. Para evitar estos problemas, se propone crear una imagen constructiva de la evaluación y pasar de un enfoque burocrático a otro más profesional, explicitar el modelo de docente ideal que orienta la evaluación y relacionar la evaluación del docente con la del centro en su conjunto, entendiendo que no es posible evaluar al profesorado sin tener en cuenta los valores del centro, su cultura y su contexto. Finalmente, recomienda que se evalúe el desempeño con el docente, no contra él, y reconoce que el gran 
reto de los sistemas de evaluación del desempeño es cómo hacer que la evaluación se convierta en mejora de la calidad.

En el caso de América Latina, Vaillant (2004) destaca que en la región se otorga un lugar especial a la antigüedad como el principal componente para que el docente pueda avanzar en una carrera profesional, y que la paradoja es que sólo se permite el ascenso a otros puestos alejando al docente del aula, lo cual tiene como consecuencia perversa el abandono de la tarea de enseñar por parte de quienes son buenos maestros. Señala que la evaluación de los docentes es casi inexistente y hay falta de incentivos para que los mejores docentes trabajen en las escuelas de contextos más desfavorecidos.

El trabajo "Estado actual de la evaluación docente en trece países de América Latina” concluye que la evaluación docente de los postulantes, de la formación inicial y del desempeño profesional no constituye una política educativa de larga data y tradición en América Latina, a pesar de que ya existen disposiciones jurídicas que sustentan la aplicación de esta política educativa. "Todavía, existen sindicatos de educadores en países latinoamericanos que mantienen una actitud de rechazo a la implementación de la evaluación docente, lo que revela que queda mucho por hacer para lograr que este tipo de política educativa tenga la viabilidad política que requiere" (Schulmeyer, 2004, p. 40). Desde esta perspectiva, la evaluación del desempeño puede contribuir de manera decisiva, sobre todo si se centra en el desarrollo profesional (idem, ibid.).

En la región, Chile es presentado como el paradigma en materia de la evaluación de los docentes, a partir de la implementación del Sistema Nacional de Evaluación del Desempeño Docente (sNed) en 1996. El SNED está ligado a incentivos monetarios que se insertan en el sistema de incentivos grupales al conjunto del establecimiento escolar. El sistema busca; por una parte, mejorar el sistema de incentivos al que están enfrentados los docentes y, por otra, generar información comparable acerca del desempeño de los establecimientos educacionales. Esta política está orientada a todos los establecimientos del país que reciben financiamiento estatal, sean administrados por los municipios (establecimientos municipales) o administrados por el sector privado (establecimientos particulares pagados). Consiste en un premio en dinero a todos los profesores de los establecimientos educativos seleccionados por su buen desempeño. Este se mide a través de los 
La regulación de la formación y el trabajo docente...

resultados académicos de los alumnos, evaluados a través de la prueba SIMCE (Sistema de Medición de la Calidad Educativa), a partir del cual se construyen grupos "homogéneos" a nivel regional para superar las dificultades de comparación de establecimientos que el SIMCE plantea. A esto se agregan indicadores de la encuesta SIMCE a padres y otros elaborados por el Ministerio de Educación. Informes de organismos internacionales lo ponderan como un mecanismo que ha permitido dar estabilidad y previsibilidad a la política educativa en el largo plazo (Stein, 2006). Sin embargo, la implementación de este tipo de enfoque sigue siendo fuertemente dogmática y acrítica, tal como se evidencia en la conclusión de uno de los estudios: "A pesar de que hay escasas evaluaciones de sus impactos, las perspectivas que ofrece este esquema han llevado a considerarlo como una alternativa innovadora a incorporar en la agenda de las políticas educativas" (Mizala y Romaguera, 2004, p. 395).

El caso de México muestra un modelo algo distinto. En su etapa inicial, en 1993, el programa Carrera Magisterial estableció que el desempeño sería solo uno de varios criterios para determinar los aumentos salariales de los docentes, y asignó al desempeño una ponderación de 35 puntos de una escala de 100, siendo los criterios de antigüedad y formación académica los que seguirían teniendo mayor peso. Aunque el peso relativo del desempeño ha ido aumentando progresivamente desde entonces, hasta ahora el programa es administrado por un comité conjunto en donde la de Educación Pública (SEP) y el Sindicato Nacional de Trabajadores de la Educación (SNTE) tienen una representación equitativa. A lo largo de los años, Carrera Magisterial ha beneficiado a más de 700 mil docentes. En vista de su enorme magnitud, y puesto que otorga aumentos salariales permanentes en lugar de primas de una sola vez, se asemeja más a un programa de prestaciones sociales que a un programa de incentivos propiamente dicho. Hasta 2001, el gobierno federal asignaba un monto a cada estado y se encomendaba al gobierno estadual la tarea de decidir quiénes serían premiados. En la práctica, los gobiernos estaduales han asignado el máximo número de premios que permiten los fondos, empezando por los docentes en los tramos superiores de la escala salarial. En consecuencia, el límite que determina quiénes son premiados y quiénes no no ha constituido una referencia para el desempeño, sino que ha dependido de la disponibilidad de fondos de cada estado en un ejercicio determinado. El considerable control que ejerce el SNTE sobre las administraciones de educación estaduales 
ha garantizado que, en la práctica, la implementación de este mecanismo esté en manos del sindicato (Stein, 2006). Sin embargo, el informe "Análisis del sistema de evaluación y del impacto del programa de estímulos docentes Carrera Magisterial en México", elaborado para el consorcio internacional RAND Education y publicado en el 2007, concluye que a pesar de que el país ha dedicado un monto importante de recursos (un promedio de 400 millones de pesos por año desde 1998 a la fecha), el programa de carrera magisterial ha tenido un "nulo o poco impacto" en los resultados de aprovechamiento escolar.

Un cambio de perspectiva respecto del desarrollo profesional docente desplaza el eje de discusión del tema de los incentivos y de la evaluación externa. Un informe reciente, elaborado en el marco del PREAL, propone construir un "entorno profesional" que atraiga no sólo para el ingreso a la carrera docente, sino para la permanencia y el progreso en ella. Entre los componentes de una reformulación de la carrera en la perspectiva del desarrollo profesional encontramos: asignación de nuevas funciones calificadas a los docentes que ya tienen cargos en el sistema; planes de mejoramiento profesional que permitan a cada docente elaborar un proyecto de formación y desarrollo de largo alcance, adoptando como criterios las disciplinas que le interesan y los conocimientos que necesita reforzar o adquirir; estímulos a la realización de estudios superiores en el área de educación; otorgamiento de año sabático; realización de pasantías en otras escuelas, en una función similar a la que se cumple en la propia, y establecimiento de sistemas de mentorazgo, que destacan a docentes calificados y de reconocida trayectoria para el acompañamiento de maestros y profesores noveles en sus primeros desempeños laborales, con una asignación económica especial (Terigi, 2006).

En algunos países, las acciones de resistencia desarrolladas por los sindicatos docentes, impidieron el avance de las políticas de evaluación de los docentes. Como respuesta a las políticas orientadas por el modelo del Estado evaluador, han comenzado a gestarse en América Latina nuevos modos de organización de los educadores, basados en la conformación de redes que permiten realizar intercambios y producciones colectivas de conocimientos acerca de las propias prácticas, a partir de un proceso de participación protagónica. Entre estas experiencias se encuentran la Red de Cualificación de Educadores en Ejercicio, en Colombia; el Programa de Transformación de la Educación 
Básica desde la Escuela, en México; la Red de Docentes que Hacen Investigación Educativa, en Argentina; la Red de Investigación en la Escuela, en Brasil, y las redes que abordan la problemática de la lengua materna, en los casos de Chile y Perú. Estos nuevos movimientos son alternativas de formación, cuyo factor común es el desarrollo de proyectos de innovación de la práctica docente e investigación educativa, mediante la participación colectiva de los educadores en sus propias aulas y en la creación de redes a nivel local, nacional y regional. Estas experiencias parten de considerar los saberes de los educadores y replantean los modos tradicionales y característicos del modelo hegemónico, en cuanto al papel de las instituciones formadoras, los vínculos entre éstas y las escuelas, las condiciones de trabajo, el papel de la investigación en las instituciones de formación docente posterior y fundamentalmente, los modos verticalistas con que hasta ahora se impulsaron todas las reformas educativas (Cardelli y Duhalde, s/f).

\section{Reflexiones finales}

La construcción de la "agenda educativa" de la Región responde a un mecanismo a través del cual a la crisis del modelo burocrático de la escuela pública se opone como alternativa otro modelo, basado en la racionalidad del mercado, presentado como el único camino posible que puede lograr una mejora de la calidad de la educación, a pesar de que los propios estudios presentan evidencias que contradicen las propuestas que terminan sosteniendo. Es por ello que se busca imponer una única agenda más allá de los contextos de su implementación.

Al docente, en tanto sujeto colectivo, se lo señala en buena medida como responsable por la crisis del sistema burocrático. Al mismo tiempo, al docente, en tanto individuo, se lo responsabiliza por los resultados de los cambios a implementar. La acción colectiva busca reemplazarse por respuestas de carácter más individual al tiempo que se desplaza la responsabilidad por la garantía de los derechos de los ciudadanos del Estado a los sujetos y a las instituciones.

Por otra parte, es interesante ver cómo la imposición de los temas de la agenda parte de la necesidad de los Estados de dar respuesta a la crisis de sus sistemas educativos. Continúa con una serie de consultores y expertos que realizan estudios e informes (financiados en su 
gran mayoría por los organismos multilaterales de crédito), recomendando la implementación de políticas basadas en la lógica del mercado; sigue con la contratación de esos expertos por parte de los ministerios de educación de los países para aplicar los planes de reforma financiados por los mismos organismos. A su vez, estos estudios en general basan el análisis de las "experiencias exitosas" en los propios informes que realizan los responsables ministeriales que las diseñan e implementan, tal como se desprende de la bibliografía y de las fuentes citadas en estos informes.

Las relaciones causa-efecto que en forma lineal establecen entre los docentes y los resultados de los aprendizajes, desde un enfoque sectorial de la educación, y, en consecuencia, las medidas que proponen terminan encerrando el problema de la regulación de los sistemas educativos dentro de un cerco que en modo alguno puede constituirse en una alternativa superadora, en un sentido democratizador, frente a la crisis de los modos tradicionales de regulación del sistema. Las falencias del modelo de regulación burocrático-profesional, que sirvió de base a la expansión de la escuela pública en el pasado, obligan a buscar nuevas modalidades de regulación que permitan recrear la escuela como espacio público de decisión colectiva, en base a la igualdad y el respeto por la diversidad (Barroso, 2005). Las nuevas regulaciones, que resultan de una particular combinación entre formas de control dirigidas al autocontrol con mecanismos externos de regulación y que buscan orientar las políticas educativas de la Región, están muy lejos de dar respuesta a este desafío.

\section{Recebido em maio de 2007 e aprovado em julho de 2007.}

\section{Notas}

1. Entre algunos de los autores que analizan estas transformaciones, destacamos los aportes de François Dubet, Joan Subirats, Luc Boltansky y Eve Chiapello, Manuel Castells, Richard Sennet, Robert Castel, Samir Amin, Ulrich Beck, Zygmunt Bauman. Para la mirada desde América Latina: Alberto Minujin, Atilio Borón, Boaventura de Souza Santos, Maristella Svampa; Norbert Lechner, Norberto Ianni, Oscar Oszlak; Renato Ortiz; Ricardo Antunes, entre muchos otros.

2. Bolivar (2004) desarrolla esta idea para el análisis de los discursos y propuestas de autonomía escolar

3. El Grupo de Trabajo cuenta con un comité de seis miembros y con el apoyo de una red de profesionales pertenecientes a universidades e institutos de formación docente, centros de investigación, asociaciones de docentes, Ministerios de Educación, organismos 
La regulación de la formación y el trabajo docente...

internacionales, organizaciones de la sociedad civil y del mundo empresarial (http:// www.preal.org/GTD)

4. Los miembros del Consejo Consultivo de PREAL "suscriben Informes sobre el Progreso Educativo Regional donde se examina el estado de la situación y se formulan recomendaciones para resolver los problemas que la aquejan. Se trata de informes cortos, directos y en lenguaje sencillo, orientados al perfeccionamiento de políticas, donde se hace un llamado a actores sociales estratégicos a involucrarse más activamente en el cambio educativo". PREAL "promueve la participación de diversos actores sociales en el desarrollo y perfeccionamiento de políticas y estimula la búsqueda de acuerdos nacionales para mejorar la calidad, equidad y eficacia de los sistemas de educación. Para ello, trabaja a través de una red de contrapartes nacionales compuesta por organizaciones de la sociedad civil, entre las que participan, o han participado, centros dedicados a la investigación social y al análisis y formulación de políticas públicas, fundaciones de origen empresarial, corporaciones privadas, centros académicos independientes, organizaciones gremiales y civiles, medios de comunicación y organizaciones eclesiásticas" (http://www.preal.org).

\section{Referencias bibliográficas}

BANCO MUNDIAL. Prioridades y estrategias para la educación: examen del Banco Mundial. Washington, DC, 1996.

BARROSO, J. La autonomía de las escuelas en el contexto de cambio de los modos de regulación de las políticas y de la acción educativa: el caso portugués. Revista de Educación, Madrid, n. 333, ene./abr. 2004.

BARROSO, J. O Estado, a educação e a regulação das políticas públicas. Educação \& Sociedade, Campinas, v. 26, n. 92, p. 725-751, out. 2005.

BECK, U. ¿Qué es la globalización?: falacias del globalismo, respuestas a la globalización. Buenos Aires: Paidós, 2004.

BOLIVAR, A. La autonomía de los centros escolares en España: entre declaraciones discursivas y prácticas sobrerreguladas. Revista de Educación, Madrid, n. 333, ene./abr. 2004.

BURBULES, N.; DENSMORE, K. Los límites de la profesionalización en la docencia. Educación y Sociedad, Madrid, n. 11, p. 67-83, 1992.

COMISIÓN ECONÓMICA PARA AMERICA LATINA Y EL CARIBE (CEPAL). Educación y conocimiento: eje de la transformación productiva con equidad. Santiago de Chile: CEPAL/UNESCO, 1992. 
CROZIER, M. La transición del paradigma burocrático a una cultura de gestión pública. Reforma y Democracia: revista del ClAD, Caracas, n. 7, ene. 1997.

DALE, R. Los efectos de la globalización en la política nacional: un análisis de los mecanismos. In: Bonal, X.; Tarabini, A.; Verger, A. (Ed.). Globalización y educación: textos fundamentales. Buenos Aires: Miño y Dávila, 2007. (no prelo)

DE MARINIS, P. 16 comentarios sobre la(s) sociología(s) y la(s) comunidad(es). Papeles del CEIC, Bilbao, n. 15, p. 1-39, 2005. Disponível em: <http://www.ehu.es/CEIC/papeles/15.pdf >

DELEUZE G. Postdata sobre las sociedades de control. In: Ferrer, C. (Comp.). El lenguaje literario. Montevideo: Nordan, 1991. t. 2.

DIAZ, H.; SAAVEDRA, J. La carrera de maestros: factores institucionales, incentivos económicos y desempeño. Washington, DC: BID, 2000.

FELDFEBER, M. Las políticas de formación docente en el contexto de la reforma educativa. Revista del Instituto de Investigaciones en Ciencias de la Educación, Buenos Aires, v. 8, n. 15, dic. 1999.

FELDFEBER, M.; OlIVEIRA, D.A. (Comp.). Politicas educativas y trabajo docente: nuevas regulaciones, ¿nuevos sujetos? Buenos Aires: NOVEDUC, 2006.

FREITAS, H.L. Certificação docente e formação do educador: regulação e desprofissionalização. Educação \& Sociedade, Campinas, v. 24, n. 85, p. 1.095-1.124, dez. 2003.

LANG, V. La construcción social de las identidades profesionales de los docentes en Francia: enfoques históricos y sociológicos. In: TENTI Fanfani, E. (Comp.). El oficio de docente: vocación, trabajo y profesión en el siglo XXI. Buenos Aires: Siglo Veintiuno, 2006.

LIANG, X. Teacher pay in 12 Latin American countries: how does teacher pay compare to other professions, what determines teacher pay, and who are the teachers? Washington, DC: World Bank, 1999. (World Bank LCSHD paper series n. 49)

MIZALA, A.; ROMAGUERA, P. El sistema nacional de evaluación del desempeño docente (SNed). In: Maestros en América Latina: 
La regulación de la formación y el trabajo docente...

nuevas perspectivas sobre su formación y desempeño. Santiago de Chile: PREAL-BID, 2004.

MORGENSTERN DE FINKEL, S. La crisis de la sociedad salarial y las políticas de formación de la fuerza de trabajo; ponencia presentada en el Congreso Latinoamericano de Sociología del Trabajo, Buenos Aires, 17 al 19 de mayo de 2000. Revista Latinoamericana de Estudios del Trabajo, México, v. 6, n. 11, p. 117-148, 2000.

MOURA CASTRO, C.; IOSCHPE, G. La remuneración de los maestros en América Latina: ¿Es baja? ¿Afecta la calidad de la enseñanza? Santiago de Chile: Preal, 2007. (Documento, 37)

MURILLO TORRECILLA, F.J. (Coord.). Evaluación del desempeño y carrera profesional docente: una panorámica de América y Europa. Santiago de Chile: orealc/unesco, 2006.

OLIVEIRA, D.A. Regulação das políticas educacionais na América Latina e suas consequências para os trabalhadores docentes. Educação \& Sociedade, Campinas, v. 26, n. 92, p. 753-775, out. 2005.

PREALC/OREALC-UNESCO. Gestión de la Educación en América Latina y el Caribe, ¿vamos por un buen camino? Documento para la discusión, Seminario de Alto Nivel Cepal-unesco, San Juan, Puerto Rico, 30 jun. 2004.

POPKEWITZ, T.; PEREYRA, M. Estudio comparado de las prácticas contemporáneas de reforma de la formación del profesorado en ocho países: configuración de la problemática y construcción de una metodología comparativa. In: Popkewitz, T. (Comp.). Modelos de poder y regulación social en pedagogía: crítica comparada de las reformas contemporáneas de la formación del profesorado. Barcelona: PomaresCorredor, 1994.

SCHULMEYER, A. Estado actual de la evaluación docente en trece paises de América Latina. Trabajo presentado a la Conferencia Regional "El Desempeño de los Maestros en América Latina y el Caribe: nuevas prioridades", Brasilia, DF, 10-12 jul. 2002.

SENNETT, R. La corrosión del carácter: las consecuencias personales del trabajo en el nuevo capitalismo. Barcelona: Anagrama, 2000. 
STEIN, E. et al. (Coord.). La politica de las politicas públicas: progreso económico y social en América Latina; informe 2006. Washington, DC: BID, 2006.

TENTI FANFANI, E. Algunas dimensiones de la profesionalización de los docentes: representaciones y temas de la agenda política. Revista PRELAC, Chile, n. 0, ago. 2004.

TENTI FANFANI, E. La condición docente: datos para el análisis comparado Argentina, Brasil, Perú y Uruguay. Buenos Aires: Siglo Veintiuno, 2005.

TENTI FANFANI, E. Profesionalización docente: consideraciones sociológica In: Tenti Fanfani, E. (Comp.). El oficio de docente: vocación, trabajo y profesión en el siglo Xxi. Buenos Aires: Siglo Veintiuno, 2006.

TERIGI, F. Desarrollo profesional continuo y carrera docente en América Latina: resumen ejecutivo. [S.1.]: PREAL, 2006.

TORRES, R.M. Justicia educativa y justicia económica: 12 tesis pare el cambio educativo. [s.l.]: Fe y Alegría; Entreculturas, 2005.

UNESCO. Formación docente: un aporte a la discusión; la experiencia de algunos países Santiago de Chile: unesco/orealc, 2002.

VAILLANT, D. Construcción de la profesión docente en América Latina: tendencias, temas y debates. Santiago de Chile: PrEAL, 2004. (Documento, 31)

VAILLANT, D.; ROSSEL, C. (Ed.). Maestros de escuelas básicas en América Latina: hacia una radiografía de la profesión. Santiago de Chile: PREAL, 2006.

VEGAS, E.; PRITCHETT, L.; EXPERTON, W. Cómo atraer y retener docentes calificados en la Argentina: impacto del nivel y estructura de la remuneración. Washington, DC: Banco Mundial, 1999.

WHITTY, G.; POWER, S.; HALPIN, D. La escuela, el Estado y el mercado. Madrid: Morata; Paideia, 1999. 